\title{
PERPETUAL LEAPFROGGING IN BERTRAND DUOPOLY Emanuele Giovannetti*
}

*Dipartimento di Economia Pubblica, Universita' degli studi di Roma "La Sapienza", and Univeristy of Cambridge, U.K. I would like to thank Jayasri Dutta for her help and suggestions at many different stages of this work, Vincenzo Denicolo', Ramon Marimon, Colin Rowat, Emanuela Sciubba and Jean Pierre Vidal for useful comments. The usual disclaimer applies. 


\section{Introduction}

In this paper we focus on the intertemporal aspects of technological adoption. We consider a simplified model: firms produce an homogeneous good and adoption decisions concern a cost reducing technology. We focus on the issue of industrial leadership reversal.

Imagine an industry facing a sequence of cost reducing innovations; the appearance of newer generations of PC processors provides a good example of the sort of improvements we have in mind.

Individual firms can upgrade by adopting the most recent improvement. This improvement comes at a cost, for example, of installing the new processors. Will firms choose to make these costly adoptions? How do adoption rates depend on the product market competition? Which adoption patterns will be sustainable in equilibrium? Will these adoption strategies reduce aggressive competition?

We consider a duopoly where firms set prices, i.e. there is Bertrand competition in the product market. In an intertemporal, infinite horizon setting, firms can adopt alternative and complicated dynamic adoption strategies. We study Markov Perfect equilibria (MPE's) and in addition restrict attention to equilibria with relatively simple but economically relevant patterns. These are: 1) Alternating adoptions and 2) Increasing asymmetry. An increasing asymmetry pattern of adoptions is such that the firm with the lowest unit cost adopts, while the high cost firm does not, so 
that existing cost asymmetry is reinforced. An alternating adoptions pattern is such that the firm with high unit cost adopts, while the low cost firm does not, existing cost asymmetry is reversed and leapfrogging takes place.

We find that if adoption is profitable at a given date, and the price elasticity of demand is greater than, or equal to, one, then no asymmetry can be absorbing and technological adoption goes on forever through an infinite sequence of leapfroggings. For this case we characterize the adoption cost region where a pattern of alternating adoptions is an MPE. In this setting increasing asymmetry is never an MPE.

Perpetual leapfrogging emerges therefore as a set of simple adoption strategies allowing implicit and sustainable coordination between two firms. Such coordination helps avoiding the most aggressive aspects of duopolistic price competition. Only with high price elasticity, and a market size large enough compared to adoption costs, this goes on forever ${ }^{1}$.

If adoption is profitable at a given date but the elasticity of demand is below one, there is a date in which the adoption process will stop. Alternating adoptions up to this date is an MPE for a range of adoption costs. Increasing asymmetry can also be an equilibrium in this case, but under very restrictive conditions.

In an oligopolistic industry demand conditions play an essential role in determining both the continuation or the end of the technological adoption 
and the identity of the adopters. When adoption continues, long run technological improvements are only made by high cost firms, which emerge as the engine of productivity growth. This is mainly due to the nature of the incentives for the adoption of a new technology under Bertand competition. For any downward sloping demand function the increments in period profits, due to the adoption of a new cost reducing technology, are larger for the follower than for the leader, because market demand when the follower adopts is higher than when the leader adopts. In this last case there is, indeed, a higher equilibrium price ${ }^{2}$.

With isoelastic demand functions the value of the elasticity determines whether the adoption process will go on forever or not. With more general demand functions one would need to study the limit behaviour of the profits' increments, due to adoption, when the equilibrium price tends to zero. Only if demand grows proportionally faster than the decrease of the price-cost margin, adoption of new technologies can go on forever.

After a brief review of some related literature, the remainder of the paper is organized as follows: in section 2 we describe the model industry: market demand, technology evolution and costs, and firms' decision sets and objective functions. In section 3 we analyse adoption decisions in a Bertrand duopoly, first with myopic firms and then with discounting. Finally section 4 contains the conclusions of the paper. All the proofs are 
contained in the appendix.

\subsection{Related literature}

Most of the literature studying innovation decisions in a strategic context has considered the problem of invention. The prototype model is the patent race, Lee and Wilde (1980). Players involved in the race have often distinct roles $^{3}$, an incumbent monopolist and a challenger, or two firms with different initial unit costs.

The conclusions about which equilibrium prevails in the incumbent/ challenger case have been twofold: Gilbert and Newbery (1982) proved that the monopolist preempts the challenger and wins the patent because of the dissipation of industry profits associated with a less concentrated market structure. Reinganum (1983) shows that, when winning the race gives sufficiently high post innovation profits, the challenger is more likely to win because of the profit replacement effect.

A testable implication of Reinganum's result, similar to our conclusions, is that "challengers contribute disproportionately more large innovations". Considering a finite sequence of drastic innovations, Reinganum (1985) obtains an industry with turnover of technological leadership.

Analyzing a finite sequence of bidding games for non-drastic innovations Vickers (1986) shows that under Bertrand competition persistent dominance is the only possible equilibrium. 
Fudenberg et al. (1983) studied a race made by a sequence of bids to obtain a patent. In their model the follower has a chance to leapfrog only if he can overtake the leader in one step, but there is a maximum level of cumulated asymmetry leading the follower to drop out of the contest, while Harris and Vickers (1985) and (1987), by assuming that the follower has to go through all the steps of the existing gap with the leader, find that the identity of the winner depends on the distance between the state of each firm and the finishing line of the race.

A setting closer to ours is one in which firms race for a sequence of innovations. Beath, Katsoulacos and Ulph (1994) consider both the case in which the follower can compete for the "state of the art technology" (they call this setting a leapfrog technology) and the case in which each firm has to discover every technology step by step (they call this case a catch-up technology).

By running stochastic simulations they observe, for a Bertrand duopoly, the emergence of persistent dominance both under catch- up and leapfrog technology.

The question of whether asymmetries between firms tend to increase or decrease during a multiple stage race has been addressed by Budd, Harris and Vickers (1993). In their analysis firm profit flows depend on the gap between their technology levels and effort rates are functions of the level of 
technological asymmetry. The solution concept employed for the analysis of the resulting dynamic stochastic game is a subgame perfect equilibrium in stationary Markov strategies.

A second strand of literature emphasizes the demand side of the innovative activity. Strategic models of adoption focus on the timing of adoption of an innovation which is otherwise available to all firms in the industry. For a survey of the literature on the timing of adoption in a strategic context see Reinganum (1989), for more recent contributions which use the Markov Perfect Equilibrium concept see Kapur (1994) and (1995).

In this paper we focus on adoption of cost reducing technologies instead than on invention. In this we follow Jovanovic (1997) who emphasized that considering learning-based adoption costs, schooling and on-the-job training, and applied R\&D spending as total adoption costs these:

"amount to at least 10-15 \% of U.S. output...adoption costs outweigh invention costs roughly 20 or 30 to 1 . In LDCs the ratio must be astronomical". Jovanovic (1997 page 332).

\section{The Model}

We introduce a specification of the demand and technology conditions 
that we will maintain throughout the paper.

- Time $t$ is discrete.

- There is an isoelastic demand function:

$$
D\left(p_{t}\right)=A p_{t}^{-\gamma}
$$

where $p_{t}$ is the price of one unit of the industry's homogeneous good at time $t, \gamma>0$, is the elasticity of demand, and $A$ is a parameter representing market size.

- The evolution of a cost reducing technology is described by the following deterministic

process:

$$
c_{t+1}=\left\{\begin{array}{cc}
\frac{c_{t}}{(1+g)} & \text { if a firm has adopted at } t-1 \\
c_{t} & \text { otherwise }
\end{array}\right.
$$

where $c_{t+1}$ is the cost of producing one unit of an homogeneous good with the state of the art technology available for adoption at time $t$ and $g>0$ is exogenously given ${ }^{4}$.

- for every firm $i$, technology adoption is a binary choice

$$
\omega_{i, t} \in\{0,1\}, \forall t
$$

and adoption costs are constant 


$$
q_{i, t}=q \omega_{i, t}
$$

with $q>0$.

- Firms' objective functions are the sum of their discounted period profits minus adoption costs:

$$
\sum_{i=0}^{\infty} \beta^{t}\left(\pi_{i, t}-q \omega_{i, t}\right)
$$

where $\beta \in[0,1)$ is a common discount factor and

$$
\pi_{i, t}=D\left(p_{t}\right)\left(p_{t}-c_{i, t}\right)
$$

represents period profits.

\subsection{Bertrand competition}

\subsubsection{Myopic equilibria}

At any date $t$, given (1) and (2), two competing firms decide whether to adopt a new technology:

$$
\omega_{i} \in\{0,1\}, i=A, B
$$

If firm $A$ has last adopted at time $t-1$ technology $c_{t}$ and firm $B$ has last adopted at time $t-k-1$, technology $c_{t-k}, k>0$, we know that $c_{A}=c_{t}<c_{B}=c_{t-k}$.

Under Bertrand competition the payoff matrix of the stage game in 
adoption is:

\begin{tabular}{|l|l|l|}
\hline$\frac{B}{A}$ & $\omega_{B, t}=0$ & $\omega_{B, t}=1$ \\
\hline$\omega_{A, t}=0$ & $A c_{t-k}^{-\gamma}\left(c_{t-k}-c_{t}\right), 0$ & $0, A c_{t}^{-\gamma}\left(c_{t}-c_{t+1}\right)-q$ \\
\hline$\omega_{A, t}=1$ & $A c_{t-k}^{-\gamma}\left(c_{t-k}-c_{t+1}\right)-q, 0$ & $-q,-q$ \\
\hline
\end{tabular}

Let us define:

- $G_{t} \equiv\left\{\omega_{A, t}=1, \omega_{B, t}=1\right\}$ as a strategy combination of global adoption;

- $F_{t} \equiv\left\{\omega_{A, t}=1, \omega_{B, t}=0\right\}$ as a strategy combination leading to forging ahead;

- $L_{t} \equiv\left\{\omega_{A, t}=0, \omega_{B, t}=1\right\} \quad$ as a strategy combination leading to leapfrogging; and,

- $S_{t} \equiv\left\{\omega_{A, t}=0, \omega_{B, t}=0\right\}$ as a strategy combination of status quo.

In the next proposition we consider the necessary and sufficient conditions for different period strategy combinations to be a Nash equilibrium of the stage game described by the payoff matrix (3).

\section{Proposition 1}

Let $\beta=0$ and $c_{A, t}=c_{t}<c_{B t}=c_{t-k}$. At any stage $t$, of the game:

a) $G_{t}$ is never an equilibrium;

b) $F_{t}$ is an equilibrium if and only if:

$$
q<\left(c_{t}-c_{t+1}\right) A c_{t-k}^{-\gamma}
$$


c) $L_{t}$ is an equilibrium if and only if:

$$
q<\left(c_{t}-c_{t+1}\right) A c_{t}^{-\gamma}
$$

and

d) $S_{t}$ is an equilibrium if and only if

$$
q \geq\left(c_{t}-c_{t+1}\right) A c_{t}^{-\gamma}
$$

From the previous proposition we can see that at any time $t^{5}$, the adoption costs space can be partitioned into three disjoint regions

$$
\begin{gathered}
Q_{1}=\left[0, K(1+g)^{\gamma(t-k)-t-1}\right) \\
Q_{2}=\left[K(1+g)^{\gamma(t-k)-t-1}, K(1+g)^{\gamma t-t-1}\right) \\
Q_{3}=\left[K(1+g)^{\gamma t-t-1}, \infty\right)
\end{gathered}
$$

with $K=A c^{1-\gamma} g$, and

$$
q \in\left\{Q_{1} \cup Q_{2} \cup Q_{3}\right\}
$$

so that, under myopic behaviour, for low adoption costs, $q \in Q_{1}$, we are in a multiple equilibria region where both leapfrogging and forging ahead are equilibria; for intermediate adoption costs, $q \in Q_{2}$, leapfrogging is the only equilibrium; for high adoption costs, $q \in Q_{3}$, status quo is the only 
equilibrium.

\subsubsection{Equilibria with discounting}

In the following we analyse sequences of adoption decisions in a Bertrand duopoly where firms have a positive discount rate $\beta$.

Starting from period $t=0$ each firm selects an infinite sequence of adoption decisions for all the future dates and states of the pay-off relevant variables. At any time, $t$, the action set of a firm is, as before, $\omega_{i, t} \in\{0,1\}$ and the previous history of play, the history of previous adoptions, defines the value of a state variable specifying the two current cost levels of firms $A$ and $B, c_{A}$, and $c_{B}$, defined by the last adoptions for each firm. The available new cost reducing technology, $c_{t+1}$, is given by the past adoption history as specified in (2).

Let us define each firm's strategy:

$$
\sigma_{i}:\left(c_{A}, c_{B}\right) \rightarrow\{0,1\}
$$

as a mapping from the set of states, of the history dependent state variables, to the set of actions $\omega_{i, t} \in\{0,1\}$. Strategies characterized in this way are known as Markov strategies. From each stage $t$ the continuation game forms a proper subgame and the state variable has a fixed value given by the previous history of adoption decisions. If we define a strategy profile composed by Markov strategies which form a Nash equilibrium for all the possible subgames starting at any value of the state variable on which the 
strategies are defined, such strategy profile would form a Markov Perfect Equilibrium $(M P E)$ of the entire intertemporal game ${ }^{6}$.

In the next sections we will focus on the parameter conditions required for specifically interesting strategy profiles (where adoption decisions follow a regular periodicity), to be MPEs of the intertemporal game.

\subsection{Alternating adoptions}

Consider the dynamic strategy profile generating a finite or infinite sequence of alternating adoptions:

$$
L_{t^{*}}=\left\{\begin{array}{cc}
\sigma_{A}\left(c_{A}, c_{B}\right)=1 & \text { if } c_{A} \geq c_{B} \text { and } c_{B}(1+g)^{-1} \geq c_{t^{*}} \\
\sigma_{A}\left(c_{A}, c_{B}\right)=0 & \text { otherwise } \\
\sigma_{B}\left(c_{A}, c_{B}\right)=1 & \text { if } c_{A}<c_{B} \text { and } c_{A}(1+g)^{-1} \geq c_{t^{*}} \\
\sigma_{B}\left(c_{A}, c_{B}\right)=0 & \text { otherwise }
\end{array}\right.
$$

This strategy profile generates the following outcome path in adoption choices:

$$
\begin{aligned}
\left\{\begin{array}{l}
\omega_{A t} \\
\omega_{B t}
\end{array}\right\} & =\left\{\begin{array}{l}
1,0,1,0,1,0, \ldots \\
0,1,0,1,0,1, \ldots
\end{array}\right\} \text { until } t^{*}-1 \\
\text { and } \omega_{A t} & =\omega_{B t}=0, \text { for } t \geq t^{*}
\end{aligned}
$$

with no halting if $t^{*}=\infty$.

Notice that :

- our strategy profile specifies that if there are symmetric initial conditions 
firm $A$ makes the first move. The parameters of the model (setting $t^{*}$ to be even or odd) determine which player moves last,

- $c_{t^{*}}$ is an absorbing technological level after which adoption stops and asymmetry is preserved,

- whether $t^{*}<\infty$ or $t^{*}=\infty$ depends on the limit behaviour of the period profits of the adopting firm associated to the strategy profile (7) as $t$ tends to infinity and, as the next lemma shows, this critical property is dependent upon the elasticity of demand.

Lemma 1

Given the expression of the adopter's period profits associated with a strategy profile of alternating adoptions:

$$
\pi_{t}=A c_{t}^{-\gamma}\left(c_{t}-c_{t+1}\right)
$$

we have that:

$$
\begin{aligned}
& \gamma>1 \Rightarrow \lim _{t \rightarrow \infty} \pi_{t}=\infty, \\
& \gamma=1 \Rightarrow \lim _{t \rightarrow \infty} \pi_{t}=\pi, \forall t \geq 0, \text { where } \pi=A \frac{g}{1+g}, \\
& 0<\gamma<1 \Rightarrow \lim _{t \rightarrow \infty} \pi_{t}=0
\end{aligned}
$$

In the following we define the set of adoption costs and discount rates such that, starting from initial symmetric cost conditions, the two duopolists will keep on leapfrogging each others by following the alternating adoption strategy profile. We consider the two cases when the demand elasticity is greater than or equal to one, and when it is between 
zero and one. As we have seen in Lemma 1 the stage game profits associated to alternating adoptions have different asymptotic behaviour in these two cases and therefore the equilibrium requirements will also differ.

Proposition 2

If $\gamma \geq 1$, the strategy profile of alternating adoptions, $L_{t^{*}}$, with

$t^{*}=\infty$, defined in (ref: $\mathrm{Lt}^{*}$ ) is an MPE for the entire game

starting in state:

$$
s_{0,0}=\left\{c_{A}=c, c_{B}=c\right\}
$$

if and only if:

$$
q \in Q_{L_{\infty}}^{\beta}(0)=\left(0, \frac{1-\beta^{2}}{(1+g)^{2(1-\gamma)}-\beta^{2}} K(1+g)^{-2 \gamma+1}\right]
$$

and

$$
\beta<\frac{1}{(1+g)^{\gamma-1}}
$$

In the next corollary we study how the alternating adoptions equilibrium region, defined in Proposition 2, and calculated from any initial date $t$, and any initial adoption gap $k$, as well as from the initial state: $s_{0,0}=\left\{c_{A}=c, c_{B}=c\right\}$, changes with the discount factor $\beta$.

Let:

$$
s_{t, t-k}^{A}=\left\{c_{A}=c_{t}, c_{B}=c_{t-k}\right\}
$$

be a state where firm $A$ has lower unit costs than firm $B$, and similarly, 


$$
s_{t, t-k}^{B}=\left\{c_{B}=c_{t}, c_{A}=c_{t-k}\right\}
$$

a state where firm $B$ has lower unit costs than firm $A$.

Corollary 1

If $\gamma \geq 1$ the adoption cost region for which alternating adoptions is an equilibrium starting at any state $s_{t, t-k}^{A}$ or $s_{t, t-k}^{B}$ :

$$
Q_{L_{\infty}}^{\beta}(t)=\left(0, \frac{1-\beta^{2}}{(1+g)^{2(1-\gamma)}-\beta^{2}} K(1+g)^{\gamma(t-2)-t+1}\right]
$$

is continuous and increasing in $\beta$, for $\beta \in[0,1)$.

Lemma 1 showed that if the elasticity of demand is between zero and one, stage period profits tend to zero under the alternating adoption strategy profile. This implies that adoption will not go on forever. To study the conditions under which a finite number of alternating adoptions is an equilibrium of the infinite horizon game we need to start by the characterization of the state/date where adoption stops.

Suppose we are in a state, $s_{t, t-k}^{A}$, where firm $A$ has lower unit costs than firm $B$. We define absorbing asymmetry as a strategy profile for the subgame starting at state $s_{t, t-k}^{A}$, such that:

$$
\omega_{A, t+i}=\omega_{B, t+i}=0, \forall i=0,1,2, \ldots
$$

Proposition 3 
If

$$
0<\gamma<1
$$

a strategy profile of absorbing asymmetry is a Markov Perfect

Equilibrium for the game starting at any state

if and only if

$$
s_{t, t-k}^{A}=\left\{c_{A}=c_{t}, c_{B}=c_{t-k}\right\}
$$

$$
q \in Q_{A A}^{\beta}(t)=\left[\frac{K(1+g)^{t(\gamma-1)-1}}{(1-\beta)}, \infty\right)
$$

Two points are worth noticing: first in deriving the necessary and sufficient cost condition for a state of asymmetry to be absorbing ${ }^{7}$ we find that only the follower's deviation matters and, second, the amount of the previously cumulated difference expressed by $k$, does not play any role in the equilibrium condition. This is explained by the fact that the profit for the follower is zero given the Bertrand structure, irrespective of the number of periods it has been lagging behind, expressed by $k$.

We are finally ready to study the conditions under which a strategy profile of alternating adoptions is an equilibrium when $0<\gamma<1$. In this case we have seen that there is a finite number of adoptions followed by absorbing asymmetry. In the following lemma we characterize the date $t^{*}$ from which adoption stops.

\section{Lemma 2}




$$
\begin{aligned}
& \text { If } 0<\gamma<1 \text { there is a date } \\
& \qquad t^{*}=\left\{\min t \in\{0,1, \ldots, \infty\}: t>\frac{\ln \left(\left(\frac{q}{K}(1+g)(1-\beta)\right)\right)}{\ln (1+g)(\gamma-1)}\right\}
\end{aligned}
$$

such that for any $t \geq t^{*}$ nobody adopts if the history of the game up to $t^{*}$ has been generated by the strategy profile of alternating adoption, $L_{t^{*}}$ defined by (7).

In the next proposition we find a sufficient condition for alternating adoptions to be an MPE when $0<\gamma<1$.

Proposition 4

For $0<\gamma<1$ the strategy profile:

$$
L_{t^{*}}: S \rightarrow\{0,1\}^{2}
$$

defined by (7), for $t^{*}$ given by (11), is an MPE if

$$
\frac{\beta}{(1-\beta)}<\frac{1}{(1+g)^{\gamma-1}}
$$

and 


$$
q \in Q_{L_{t^{*}}}^{\beta}=\left(\frac{K(1+g)^{\gamma t^{*}-t^{*}-1}}{1-\beta}, \frac{K(1+g)^{\gamma t^{*}-t^{*}-\gamma}}{1-\beta}\right]
$$

Note that for $\beta \rightarrow 0$ condition (12) is always satisfied:

$$
\lim _{\beta \rightarrow 0} \frac{\beta}{(1-\beta)}=0<\frac{1}{(1+g)^{\gamma-1}}
$$

\subsection{Increasing asymmetry}

The last pattern of adoptions we consider is one leading to increasing asymmetry between the two firms. Consider the following strategy profile:

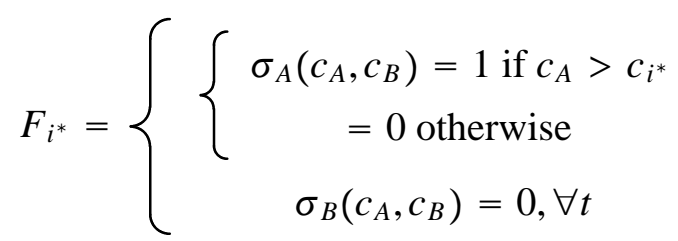

This implies that the outcome path will be:

$$
\begin{aligned}
& \begin{array}{l}
\omega_{A, t} \\
\omega_{B, t}
\end{array}=\left\{\begin{array}{l}
\{1,1,1,1,1,1,1\} \\
\{0,0,0,0,0,0,0\}
\end{array}\right\} \text { until } t=i^{*}-1 \\
& \omega_{A, t}=\omega_{B, t}=0, \text { for } t \geq i^{*}
\end{aligned}
$$

with no halting if $i^{*}=\infty$.

In the next proposition we will see that the conditions for this strategy profile leading to increasing asymmetry between the duopolists are very restrictive. Furthermore the number of periods in which asymmetry 
cumulates can not be longer than the inverse of the demand elasticity, which therefore has to be smaller than one.

\section{Proposition 5}

For $\gamma<1$, the strategy profile $F_{i^{*}}: S \rightarrow\{0,1\}^{2}$ defined by (14) is a MPE for the entire game starting from the state

$$
s_{0,0}=\left\{c_{A}=c, c_{B}=c\right\}
$$

if and only if :

$$
q \in Q_{F_{i^{*}}}^{\beta}\left(s_{0,0}\right)=\left(\frac{K(1+g)^{(\gamma-1) i^{*}-1}}{1-\beta}, \frac{K(1+g)^{-i^{*}}}{1-\beta}\right]
$$

and $i^{*}<\frac{1}{\gamma}$.

Note that $0<\gamma<1$ is a necessary condition for the strategy profile of increasing asymmetry to be an MPE of the game.

\section{Remark}

By comparing the equilibrium regions for alternating adoptions up to a finite date, as defined in Propositions 4, and for increasing asymmetry, as seen in Proposition 5, it is easy to see that, when $0<\gamma<1$, the two equilibria may co-exist and when increasing asymmetry is an equilibrium alternating adoptions is an equilibrium too.

The intuition of this result can be extended for more general downward 
sloping demand functions. The profit's increment for an adopting firm when the other is not adopting are indeed given by the product of two components:

- the difference, due to adoption, in the equilibrium price-cost margin, which under Bertrand competition, is the same, $\left(c_{t}-c_{t+1}\right)$, if the adopter is the present leader or follower and,

- the quantity demanded at the new equilibrium price. This last quantity is always larger if the follower is the adopter. The adopting follower, when becomes the new leader, will indeed charge a price equal to the unit cost of the previous leader, while the adopting leader will charge a price equal to the, unchanged, unit cost of the follower. By definition the leader has a lower unit cost, so that the previous period follower will charge a lower price and have, through a demand effect, a higher incentive to adopt the new technology.

\section{Conclusions}

In this paper we analysed firm adoption decisions about an infinite sequence of innovations. In a Bertrand duopoly with myopic firms we characterized the cost regions leading to forging ahead, leapfrogging and status quo. For low adoption costs both, forging ahead and leapfrogging are equilibria, for intermediate costs leapfrogging is the unique equilibrium and for higher costs status quo is the only equilibrium. These equilibrium regions change with the evolution of the cost reducing technology if the 
elasticity of the market demand is not equal to one. When the elasticity is below one both the forging ahead and the leapfrogging equilibria disappear and technological adoption stops, while if the demand elasticity is greater than one, adoption, once started, will never stop.

With discounting we analysed the conditions under which a strategy profile of alternating adoptions, inducing perpetual leapfrogging, is sustainable as a Markov Perfect Equilibrium. Two cases again arise: when the demand elasticity is greater than or equal to one, an alternating adoption strategy profile can go on forever.

When the demand elasticity is below one, alternating adoptions stop at an endogenously derived terminal date and the last adopter remains the leader in the industry.

Finally we considered a strategy of increasing asymmetry in which the leader adopts for a finite number of periods and the follower does not. When the elasticity of the demand is less than one we have that the maximum cumulated asymmetry is bounded above by the inverse of the demand elasticity, while if the demand elasticity is greater than one increasing asymmetry is never a Markov Perfect Equilibrium.

In conclusion when considering an infinite sequence of adoption decisions, the resulting technological dynamics is determined by the interaction of demand factors (both the elasticity and the size of the 
market), adoption costs, pace of technical progress and the discount rate. When, given these parameters, there are incentives to adopt we might expect the challengers/follower firms to account for the majority of the innovative activity. 


\section{Appendix}

Proof of Proposition 1

These are just the Nash equilibria of the game described by the Matrix (3).

\section{Proof of Lemma 1}

This is obvious by taking the limit of the increments in period profits in expression (8).

Proof of Proposition 2

We prove Markov perfection in two steps: first we characterize, in Lemma A1, the necessary and sufficient conditions for an alternating adoptions strategy profile, $L_{t^{*}}$, as defined in (7) to be a Nash equilibrium in Markov strategies for the subgames starting from any given state of technological asymmetry, when $\gamma \geq 1$, and then we show that if $L_{t^{*}}$ is a Nash Equilibrium from the origin of the game then it is an equilibrium starting from any other subgame.

\section{Lemma A1}

Let the strategy profile

$$
L_{t^{*}}: S \rightarrow\{0,1\}^{2}
$$

be defined by (7), where $S$ is the set of all possible payoff relevant states, then: for $\gamma \geq 1, L_{t^{*}}$ is a Nash equilibrium in Markov strategies with $t^{*}=\infty$, for all the subgames starting from any state : 


$$
s_{t, t-k}^{A}=\left\{c_{A}=c_{t}, c_{B}=c_{t-k}\right\}
$$

or

$$
\begin{gathered}
s_{t, t-k}^{B}=\left\{c_{B}=c_{t}, c_{A}=c_{t-k}\right\} \\
\forall t \geq 0, k \leq t
\end{gathered}
$$

if and only if :

$$
\beta<\frac{1}{(1+g)^{\gamma-1}}
$$

and

$$
q \in Q_{L_{\infty}}^{\beta}(t)=\left(0, \frac{1-\beta^{2}}{(1+g)^{2(1-\gamma)}-\beta^{2}} K(1+g)^{\gamma(t-2)-t+1}\right]
$$

\section{Proof of Lemma A1}

At any $t$, define:

- $\hat{V}_{t, i}$ as the value to firm $i$ of following (the prescription of) strategy $L_{t^{*}}$ and

- $\tilde{V}_{t, i}$ as the value to firm $i$ of a one period deviation from strategy $L_{t^{*}}$.

When firm $A$ is the current leader and the state of the system is:

$$
s_{t, t-k}^{A}=\left\{c_{A}=c_{t}, c_{B}=c_{t-k}\right\}
$$

the strategy profile dictates that $\omega_{A, t}=0$ and $\omega_{B, t}=1$, because $c_{t}<c_{t-k}$. 
The value of sticking to the strategy for firm $A$ in state $s_{t, t-k}^{A}$, given that firm $B$ is following the same strategy, is:

$$
\begin{aligned}
\hat{V}_{t, A}(0) & =0+\beta\left(\pi_{t+1}-q\right)+\beta^{3}\left(\pi_{t+3}-q\right)+\beta^{5}\left(\pi_{t+5}-q\right)+. .= \\
& =\frac{\beta \pi_{t+1}}{1-\left(\frac{\beta^{2}}{(1+g)^{2(1-\gamma)}}\right)}-q \frac{\beta}{1-\beta^{2}}=\beta \hat{V}_{t+1, A}(1) \equiv \beta \hat{V}_{t+1}
\end{aligned}
$$

while the value associated with a one period deviation, i.e. by adopting in period $t$ is:

$$
\tilde{V}_{t, A}(0)=-q+\beta \hat{V}_{t+1} \text {. }
$$

This implies that in state $s_{t, t-k}^{A}$ firm $A$ has no incentive to deviate from the strategy $L$ whenever there are positive adoption costs:

$$
\hat{V}_{t, A}(0)>\tilde{V}_{t, A}(0) \text { for any } q>0
$$

In the same state $s_{t, t-k}^{A}$ the value that firm $B$ derives from sticking to the strategy given that firm $A$ is following the same strategy, is:

$$
\begin{gathered}
\hat{V}_{t, B}(1)=\left(\pi_{t}-q\right)+0+\beta^{2}\left(\pi_{t+2}-q\right)+\beta^{4}\left(\pi_{t+4}-q\right)+. .= \\
=\frac{\pi_{t}=}{1-\left(\frac{\beta^{2}}{(1+g)^{2(1-\gamma)}}\right)}-\frac{q}{1-\beta^{2}}= \\
=\left(\pi_{t}-q\right)+\beta \hat{V}_{t+1, B}(0) \equiv \hat{V}_{t}
\end{gathered}
$$

while by deviating, by not adopting in spite of having higher costs, firm $B^{\prime} s$ 
value

$$
\tilde{V}_{t, B}(1)=0+\beta \max \left[\tilde{V}_{t, B}(1), \hat{V}_{t}\right]
$$

We need to prove that:

$$
\hat{V}_{t, B}(1)>\tilde{V}_{t, B}(1)
$$

if and only if the adoption cost, $q$, falls in the region:

$$
q \in Q_{L_{\infty}}^{\beta}(t)=\left(0, \frac{1-\beta^{2}}{(1+g)^{2(1-\gamma)}-\beta^{2}} K(1+g)^{\gamma(t-2)-t+1}\right] .
$$

Suppose that:

$$
\tilde{V}_{t, B}(1)>\hat{V}_{t, B}(1),
$$

from (19) we know that:

$$
\tilde{V}_{t, B}(1)=\beta \tilde{V}_{t, B}(1) \Rightarrow \tilde{V}_{t, B}(1)=0
$$

but if (20) holds:

$$
\hat{V}_{t, B}(1)=\hat{V}_{t}>0
$$

which leads to the contradiction:

$$
\hat{V}_{t, B}(1)>\tilde{V}_{t, B}(1)
$$

Let us now consider a state where firm $B$ is a leader: 


$$
s_{t, t-k}^{B}=\left\{c_{A}=c_{t-k}, c_{B}=c_{t}\right\}
$$

following the same reasoning we find that for firm $A$ :

$$
\begin{aligned}
\hat{V}_{t, A}(1) & =\left(\pi_{t}-q\right)+\beta \hat{V}_{t+1, A}(0)= \\
& =\frac{\pi_{t}}{1-\left(\frac{\beta^{2}}{(1+g)^{2(1-\gamma)}}\right)}-\frac{q}{1-\beta^{2}} \equiv \hat{V}_{t} \\
& \tilde{V}_{t, A}(1)=0+\beta \max \left[\tilde{V}_{t, A}(1), \hat{V}_{t}\right]
\end{aligned}
$$

and

$$
\hat{V}_{t, A}(1)>\tilde{V}_{t, A}(1)
$$

again if (20) is satisfied. This is because:

$$
\hat{V}_{t, A}(1)=\hat{V}_{t, B}(1)>\tilde{V}_{t, A}(1)=\tilde{V}_{t, B}(1)
$$

while for firm $B$ we have

$$
\hat{V}_{t, B}(0)=\frac{\beta \pi_{t+1}}{1-\left(\frac{\beta^{2}}{(1+g)^{2(1-\gamma)}}\right)}-\frac{q \beta}{1-\beta^{2}}=\beta \hat{V}_{t+1}
$$

and finally:

$$
\tilde{V}_{t, B}(0)=-q+\beta\left(\hat{V}_{t+1, B}(0)\right) \equiv-q+\beta^{2} \hat{V}_{t+2}
$$

and clearly: 


$$
\hat{V}_{t, B}(0)>\tilde{V}_{t, B}(0)
$$

Finally the condition $\beta<\frac{1}{(1+g)^{\gamma-1}}$ is required for the convergence of the equilibrium values.

Having found the conditions under which the strategy profile of alternating adoptions, $L_{t^{*}}$, is a Nash equilibrium for any subgame starting at any given state of the system, we are ready to check for Markov perfection.

If (9) holds the condition for $L_{t^{*}}$ to be a Nash equilibrium is met at $t=0$,

in state

$$
s_{0,0}=\left\{c_{A}=c, c_{B}=c\right\} .
$$

Furthermore, if $\gamma \geq 1$, from (20) we can see that if the condition for $L_{t^{*}}$ to be a Nash equilibrium is met at $s_{0,0}$ then it is met at $s_{t, t-k}^{B}$ and $s_{t, t-k}^{A}, \forall t$ and $\forall k<t$. This is because the upper bound of the cost region $Q_{L_{\infty}}^{\beta}(t)$ is increasing in $t$.

\section{Proof of Corollary 1}

The continuity of the alternating adoption region for $\beta=0$ is clear by comparing the expression of the myopic leapfrogging equilibrium region in 
(5) and the limit for $\beta \rightarrow 0$ of (20).

To see the increasing relation between $\beta$ and the region of adoption costs for which $L_{t^{*}}$ is an equilibrium we note that the partial derivative of (20) is positive for $\gamma>1$.

\section{Proof of Proposition 3}

To prove proposition 3 we start by proving Lemma A2, where we see that a given state of technological asymmetry is absorbing if and only if the firm with the higher unit cost has no incentive to adopt any number of new technologies.

\section{Lemma A2}

A strategy profile of absorbing asymmetry is a Nash equilibrium for the subgame starting from state

$$
s_{t, t-k}^{A}=\left\{c_{A}=c_{t}, c_{B}=c_{t-k}\right\}
$$

if and only if firm $B$ has no incentive to deviate from it.

\section{Proof of Lemma A2}

The values of halting the adoption once in state $s_{t, t-k}^{A}$ are:

for firm $A$, and

$$
\hat{V}_{0 B}\left(c_{t}\right)=0
$$


for firm $B$.

By deviating from the strategy profile of absorbing asymmetry, and adopting in $n$ more periods, $A^{\prime} s$ value is ${ }^{8}$ :

$$
\begin{gathered}
\tilde{V}_{n A}\left(c_{t-k}\right)=\sum_{i=0}^{n-1} \beta^{i}\left[\pi\left(c_{t+i+1}, c_{t-k}\right)-q\right]+\frac{\beta^{n} \pi\left(c_{t+n}, c_{t-k}\right)}{1-\beta} \\
=A\left[\frac{1}{1-\beta} c_{t-k}^{-\gamma+1}-c_{t-k}^{-\gamma} c_{t}\left(\frac{(1+g)^{-1}(1-\beta)+\beta^{n} g(1+g)^{-n-1}}{\left(1-\beta(1+g)^{-1}\right)(1-\beta)}\right)\right]-q \frac{1-\beta^{n}}{1-\beta}
\end{gathered}
$$

similarly, $B^{\prime} s$ value of deviating is:

$$
\begin{gathered}
\tilde{V}_{n B}\left(c_{t}\right)=\sum_{i=0}^{n-1} \beta^{i}\left[\pi\left(c_{t+i+1}, c_{t}\right)-q\right]+\frac{\beta^{n} \pi\left(c_{t+n}, c_{t}\right)}{1-\beta} \\
=A\left[\frac{1}{1-\beta} c_{t}^{-\gamma+1}-c_{t}^{-\gamma} c_{t}\left(\frac{(1+g)^{-1}(1-\beta)+\beta^{n} g(1+g)^{-n-1}}{\left(1-\beta(1+g)^{-1}\right)(1-\beta)}\right)\right]-q \frac{1-\beta^{n}}{1-\beta} .
\end{gathered}
$$

Subtracting (21) from (22) we obtain the incentive to deviate from the absorbing asymmetry strategy profile (the incentive for adopting $\mathrm{n}$ times from state $s_{t, t-k}^{A}$ ) for firm $A$ :

$$
\Delta_{n}(A)=\tilde{V}_{n A}\left(c_{t-k}\right)-\hat{V}_{0 A}\left(c_{t-k}\right)=
$$




$$
=-q \frac{1-\beta^{n}}{1-\beta}+A\left[\frac{c_{t-k}^{-\gamma} c_{t}}{1-\beta}\left(\frac{\left(1-\beta^{n}(1+g)^{-n}\right) g(1+g)^{-1}}{\left(1-\beta(1+g)^{-1}\right)}\right)\right] .
$$

Similarly, subtracting (22) from (26) we obtain the incentive for adopting $\mathrm{n}$ times from state $s_{t, t-k}^{A}$ for firm $B$ :

$$
\begin{gathered}
\Delta_{n}(B)=\tilde{V}_{n B}\left(c_{t}\right)-\hat{V}_{0 B}\left(c_{t}\right)=\tilde{V}_{n B}\left(c_{t}\right)= \\
=-q \frac{1-\beta^{n}}{1-\beta}+A\left[\frac{c_{t}^{-\gamma+1}}{1-\beta}\left(\frac{\left(1-\beta^{n}(1+g)^{-n}\right) g(1+g)^{-1}}{\left(1-\beta(1+g)^{-1}\right)}\right)\right] .
\end{gathered}
$$

Clearly:

$$
\Delta_{n}(B)>\Delta_{n}(A)
$$

since

$$
c_{t-k}>c_{t} \Rightarrow c_{t-k}^{-\gamma}<c^{-\gamma}, \forall k<t
$$

This implies that $\Delta_{n}(B)<0, \forall n \geq 1$ is a necessary and sufficient condition for $s_{t, t-k}^{A}$ to be a state of absorbing asymmetry whichever is the cost difference between leader and follower.

This leads us to the next lemma.

Lemma A3 
A strategy profile of absorbing asymmetry is a Nash equilibrium for any subgame starting from a state:

$$
s_{t, t-k}^{A}=\left\{c_{A}=c_{t}, c_{B}=c_{t-k}\right\} \forall k<t
$$

if and only if:

$$
q \in Q_{A A}^{\beta}(t)=\left[\frac{A c_{t}^{1-\gamma} g(1+g)^{-1}}{(1-\beta)}, \infty\right)
$$

\section{Proof of Lemma A3}

We have seen that a necessary and sufficient condition for an asymmetric state, $s_{t, t-k}^{A}$ to be an absorbing state is that the follower, firm $B$, has no incentive to deviate from a strategy profile in which no firm adopts a new technology.

From (28) in Lemma A2 we have seen that $B^{\prime} s$ incentive to adopt $n$ innovations given the non adoption of firm $A$, is given by:

$$
\Delta_{n}(B)=-q \frac{1-\beta^{n}}{1-\beta}+A\left(\frac{c_{t}^{-\gamma+1}}{1-\beta}\left(\frac{\left(1-\beta^{n}(1+g)^{-n}\right) g(1+g)^{-1}}{\left(1-\beta(1+g)^{-1}\right)}\right)\right)
$$

By taking the first difference of (32) one obtains $B^{\prime} s$ incentive of adopting the $(n+1)^{\text {th }}$ innovation after having adopted the previous $n$ : 


$$
\begin{aligned}
& \Delta_{n}^{2}(B) \equiv \Delta_{n+1}(B)-\Delta_{n}(B)=V_{n+1, B}\left(c_{t}\right)-V_{n, B}\left(c_{t}\right)= \\
& =q \frac{\beta^{n}}{1-\beta}(\beta-1)+A\left(\frac{c_{t}^{-\gamma+1}}{1-\beta}\right) \beta^{n} g(1+g)^{-n-1}
\end{aligned}
$$

(33) is negative if:

$$
\frac{A c_{t}^{1-\gamma} g(1+g)^{-n-1}}{(1-\beta)}<q
$$

Furthermore (34) is clearly decreasing in $n$. This implies that if the incentive to adopt the first innovation is negative, or

$$
q>\frac{A c_{t}^{1-\gamma} g(1+g)^{-1}}{(1-\beta)}
$$

there is no incentive in adopting any further innovation.

If on the contrary at $s_{t, t-k}^{A}=\left\{c_{A}=c_{t}, c_{B}=c_{t-k}\right\}$ :

$$
\exists n^{*}: \forall n<n^{*} \Rightarrow \frac{A c_{t}^{1-\gamma} g(1+g)^{-n-1}}{(1-\beta)}>q
$$

then $s_{t, t-k}^{A}$ cannot be a state of absorbing asymmetry.

We are now ready to prove Proposition 3. We have seen before that a strategy profile of absorbing asymmetry is a Nash equilibrium from any state $s_{t, t-k}^{A}$ if and only if condition (31) is satisfied. 
If $0<\gamma<1$ such strategy profile remains an equilibrium also for any subgame starting from any possible payoff relevant history, i.e. after any number of deviations, adoptions, of the leader, which from (10) define the only pay-off relevant technology level. This is because if $0<\gamma<1$ the adoption cost region, $Q_{A A}^{\beta}(t)$, is increasing in the number of deviations of the leader. Suppose for example that the leader deviated $\tau$ times from the equilibrium path of absorbing asymmetry starting from state $s_{t, t-k}^{A}$, and we are in the subgame starting from the out of equilibrium state:

$$
s_{t+\tau, t-k}^{A}=\left\{c_{A}=c_{t+\tau}, c_{B}=c_{t-k}\right\}
$$

Then from (31) we know that the necessary and sufficient condition for absorbing asymmetry to be a Nash equilibrium for the game starting at $s_{t+\tau, t-k}^{A}$ is

that

$$
q \in Q_{A A}^{\beta}(t+\tau)=\left[\frac{A c_{t+\tau}^{1-\gamma} g(1+g)^{-1}}{(1-\beta)}, \infty\right)
$$

If $0<\gamma<1$ we have that $Q_{A A}^{\beta}(t+\tau)$ includes the region $Q_{A A}^{\beta}(t)$, which in turn implies that firm $B$ has no incentive to deviate from the absorbing asymmetry strategy profile for the subgame starting at $s_{t+\tau, t-k}^{A}$ if it had no incentive to deviate from the strategy profile in state $s_{t, t-k}^{A}$. If the strategy profile of absorbing asymmetry is a Nash Equilibrium in Markov strategies starting from any possible subgame of the game starting at $s_{t, t-k}^{A}$ than it is a 
Markov Perfect equilibrium from the entire game starting at $s_{t, t-k}^{A}$.

\section{Proof of Lemma 2}

We have seen before that when $0<\gamma<1$ period profits for the adopter, along an history generated by alternating adoptions, converge to zero. This implies that, given a constant adoption cost, $q$, we can find a date $t^{*}$ from which time onwards adoption is no longer profitable.

Intertemporal profits when adopting at time $t$ for the last time in the game after a history of alternating adoption $\left(t=t^{*}-1\right)$, and with a non adopting opponent, are given by

$$
\frac{K(1+g)^{t(\gamma-1)-1}}{1-\beta}-q
$$

The last adoption date is given by the smallest $t$ such that (36) is positive. From $t^{*}$ onwards we are in a state of absorbing asymmetry.

\section{Proof of Proposition 4}

This strategy profile implies alternating adoptions for $t<t^{*}$ and absorbing asymmetry thereafter. The remaining leader would be the firm which was the latest adopter. The identity of this firm depends on whether $t^{*}$ is an even or an odd number.

\section{Incentives to deviate at time $t^{*}$}


We have seen in (31) the necessary and sufficient conditions for a given state $s_{t, t-k}^{A}$ to be a state of absorbing asymmetry. If the absorbing asymmetry state has been reached after an alternating adoption strategy profile we know that for $A$ to have adopted at time $t^{*}-1$ and for $B$ not to adopt at time $t^{*}$ it must be that:

$$
q \in\left(\frac{K(1+g)^{\gamma t^{*}-t^{*}-1}}{1-\beta}, \frac{K(1+g)^{\gamma\left(t^{*}-1\right)-t^{*}}}{1-\beta}\right]
$$

In the following, by knowing the continuation values in the two possible states:

$$
\begin{aligned}
s_{t^{*}, t^{*}-1}^{A} & =\left\{c_{A}=c_{t^{*}}, c_{B}=c_{t^{*}-1}\right\} \\
s_{t^{*}, t^{*}-1}^{B} & =\left\{c_{A}=c_{t^{*}-1}, c_{B}=c_{t^{*}}\right\}
\end{aligned}
$$

we consider, by backward induction, the incentives firms have to deviate from the strategy profile before reaching the final state at $t^{*}$.

Incentives to deviate before $t^{*}$

We start by considering the case in which $A$ is the last adopter so that the absorbing state is $s_{t^{*}, t^{*}-1}^{A}$.

\section{Firm A}

In period $t^{*}-1$, by following the strategy $L_{t^{*}}$, firm $A$ is supposed to adopt the new technology, making positive period profits: 


$$
\hat{V}_{A, t^{*}-1}(1)=\frac{K(1+g)^{\gamma\left(t^{*}-1\right)-t^{*}}}{1-\beta}-q>0
$$

by the definition of $t^{*}$

By deviating at this adoption stage (at time $t^{*}-1$ ) by not adopting, firm $A$ would make zero period profits and would remain in a state in which its technology has not changed:

$$
\tilde{V}_{A, t^{*}-1}(1)=0+\beta \max \left\{\hat{V}_{A, t^{*}-1}(1), \tilde{V}_{A, t^{*}-1}(1)\right\}=\beta \hat{V}_{A, t^{*}-1}(1)
$$

As a result of deviating firm $A$ would just postpone its intertemporal profits by adopting in the next stage and therefore does not deviate. The same reasoning applies for any state in which firm $A$ is supposed to adopt.

When Firm $A$ is in any state $s^{A}=\left\{c_{A}<c_{B}\right.$, and $\left.c_{t+1} \geq c_{t^{*}}\right\}$, in which the strategy dictates not to adopt because $A$ has lower costs, by sticking to the alternating adoption strategy firm $A$ makes zero period profits and then goes to a new state in which it will adopt (because $B$ has adopted now reverting the cost leadership). Again, by deviating in this stage and adopting, firm $A$ makes negative period profits and reaches a state in which it would adopt under the candidate equilibrium strategy, as this deviation history gives the two firms the same cost conditions. This implies that firm $A$ has no incentive to unilateral deviation at any state for $t<t^{*}$.

\section{Firm B}


Firm $B^{\prime} s$ possible deviations are more interesting because by non deviating $B$ makes zero profits from time $t^{*}-1$ onwards. Deviating at $t^{*}$ is too late; we have seen before that at $t^{*}$ the follower has no incentive to deviate because we are in a state of absorbing asymmetry. We want to see if, by deviating before $t^{*}, B$ is able to reverse the sequencing of adoptions so that it becomes the leader and dominates the industry forever.

By backwards induction when $t^{*}$ is even firm $A$ adopts at $t^{*}-1$ and $B$ does not deviate because it will face adoption costs plus zero profits forever.

At $t^{*}-2$, and in any other state in which $B$ is supposed to adopt,by deviating $B$ is only postponing profits, so it will not deviate.

At $t^{*}-3, B$ is supposed not to adopt and the value of sticking to the strategy profile is:

$$
\begin{aligned}
\hat{V}_{B, t^{*}-3}(0) & =0+\beta \max \left\{\hat{V}_{B, t^{*}-2}(1), \tilde{V}_{B, t^{*}-2}(1)\right\} \\
& =0+\beta \hat{V}_{B, t^{*}-2}(1) \\
& =0+\beta\left(K(1+g)^{\gamma\left(t^{*}-2\right)-t^{*}+1}-q\right)+\beta^{2} \max \left\{\hat{V}_{B, t^{*}-1}(0), \tilde{V}_{B, t^{*}-1}(0)\right\} \\
& =0+\beta\left(K(1+g)^{\gamma\left(t^{*}-2\right)-t^{*}+1}-q\right)+\beta^{2} 0
\end{aligned}
$$

so that:

$$
\hat{V}_{B, t^{*}-3}(0)=\beta\left(K(1+g)^{\gamma\left(t^{*}-2\right)-t^{*}+1}-q\right)
$$

If $B$ deviates at $t^{*}-3$ when it is not supposed to adopt it first pays $-q$ 
and then obtains zero period profit, because it has adopted together with $A$. Now, at time $t^{*}-2$, we are in a state where $A$ adopts because both firms have the same cost level. At time $t^{*}-1$ then $B$ finally adopts.

The value of deviating at $t^{*}-3$ is then:

$$
\begin{aligned}
\tilde{V}_{B, t^{*}-3}(0) & =-q+\beta \max \left\{\hat{V}_{B, t^{*}-2}(0), \tilde{V}_{B, t^{*}-2}(0)\right\} \\
& =-q+\beta \hat{V}_{B, t^{*}-2}(0) \\
& =-q+\beta 0+\beta^{2} \max \left\{\hat{V}_{B, t^{*}-1}(1), \tilde{V}_{B, t^{*}-1}(1)\right\} \\
& =-q+\beta 0+\beta^{2} \hat{V}_{B, t^{*}-1}(1) \\
& =-q+\beta 0+\beta^{2}\left(\frac{K(1+g)^{\gamma\left(t^{*}-1\right)-t^{*}}}{1-\beta}-q\right)
\end{aligned}
$$

so that:

$$
\tilde{V}_{B, t^{*}-3}(0)=-q+\beta^{2}\left(\frac{K(1+g)^{\gamma\left(t^{*}-1\right)-t^{*}}}{1-\beta}-q\right)
$$

By deviating at $t^{*}-3, B$ has reversed the final leadership of the industry.

For the strategy profile $L_{t^{*}}$ with $t^{*}<\infty$ to be an equilibrium from time $t=t^{*}-3$ we need

$$
\hat{V}_{B, t^{*}-3}(0)>\tilde{V}_{B, t^{*}-3}(0)
$$

by subtracting (39) from (38) we have that (40) holds whenever ${ }^{9}$ : 


$$
\frac{\beta K(1+g)^{\gamma\left(t^{*}-1\right)-t^{*}}\left((1+g)^{-\gamma+1}-\frac{\beta}{1-\beta}\right)}{(-1+\beta(1-\beta))}<q
$$

We have now two conditions on the lower bound for $q$, and we need to compare which one is more stringent between the lower bound of (37) required for having firm $A$ adopting $c_{t^{*}}$ and (41) required for the follower not to deviate from the equilibrium path before $t^{*}$. It is possible to show ${ }^{10}$ footnote that for $\gamma<1$ if

$$
\frac{\beta}{(1-\beta)}<\frac{1}{(1+g)^{\gamma-1}}
$$

condition (37) is the most stringent and therefore $L_{t^{*}}$ with $t^{*}<\infty$ is an equilibrium for:

$$
q \in Q_{L_{t^{*}}}^{\beta}=\left(\frac{K(1+g)^{\gamma t^{*}-t^{*}-1}}{1-\beta}, \frac{K(1+g)^{\gamma\left(t^{*}-1\right)-t^{*}}}{1-\beta}\right]
$$

For any stage $t^{*}-k$ where $k>3, B$ will have a smaller incentive to deviate than at $t^{*}-3$, because the payoff for reverting the leadership order is further away in time. Therefore it is sufficient to examine the condition up to $t^{*}-3^{11}$.

Proof of Proposition 5

We start by studying the incentives of firm $A$ starting from the 
beginning of the game or from a state:

$$
s_{0,0}=\left\{c_{A}=c, c_{B}=c\right\}
$$

The value of adopting $i$ innovations for firm $A$ and then stopping, given that firm $B$ does not adopt, is given by:

$$
\hat{V}(0, i)=\sum_{l=0}^{i-1} \beta^{l}\left(A c^{-\gamma}\left(c-c_{l+1}\right)-q\right)+\frac{\beta^{i}}{1-\beta} A c^{-\gamma}\left(c-c_{i}\right)
$$

while the value of adopting $i-1$ innovations is given by:

$$
\hat{V}(0, i-1)=\sum_{l=0}^{i-2} \beta^{l}\left(A c^{-\gamma}\left(c-c_{l+1}\right)-q\right)+\frac{\beta^{i-1}}{1-\beta} A c^{-\gamma}\left(c-c_{i-1}\right)
$$

By subtracting (45) from (44) we obtain the incentive of adopting the $i^{\text {th }}$ innovation for firm $A$ from state $s_{00}$ :

$$
\frac{\beta^{i-1}}{1-\beta}\left(K(1+g)^{-i}-q(1-\beta)\right)
$$

This is positive for

$$
\frac{K(1+g)^{-i}}{(1-\beta)}>q
$$

and decreases in $i$ so $c_{i^{*}}$ is determined by the maximum $i$ such that (47) 
holds or by $i$ such that

$$
\frac{K(1+g)^{-i^{*}-1}}{(1-\beta)}<q<\frac{K(1+g)^{-i^{*}}}{(1-\beta)}
$$

Next we check that at any possible history of the game, from all the possible nodes out of the equilibrium path, firm $A$ has no incentives in deviating from strategy $F_{i^{*}}$ defined in (14).

Consider any state:

$$
s_{t, k}=\left\{c_{A}=c_{t}, c_{B}=c_{k}\right\}
$$

where $t$ and $k$ are both less than or equal to $i^{*}$.

The value of adopting $i^{*}-t$ further innovations for firm $A$ is given by:

$$
\hat{V}\left(t, k, i^{*}-t\right)=\sum_{l=0}^{i^{*}-t-1} \beta^{l}\left(A c_{k}^{-\gamma}\left(c_{k}-c_{t+l+1}\right)-q\right)+\frac{\beta^{i^{*}-t}}{1-\beta} A c_{k}^{-\gamma}\left(c_{k}-c_{i^{*}}\right)
$$

while the value of adopting $i^{*}-t-1$ further innovations is given by:

$$
\hat{V}\left(t, k, i^{*}-t-1\right)=\sum_{l=0}^{i^{*}-t-2} \beta^{l}\left(A c_{k}^{-\gamma}\left(c_{k}-c_{t+l+1}\right)-q\right)+\frac{\beta^{i^{*}-t-1}}{1-\beta} A c_{k}^{-\gamma}\left(c_{k}-c_{i^{*}-1}\right)
$$

By subtracting (50) from (49) we obtain the incentive of adopting the $\left(i^{*}-t\right)^{t h}$ innovation, after having adopted the previous $t$, starting from an out of the equilibrium path node, when firm $B$ has deviated $k$ times: 


$$
\frac{\beta^{i^{*}-t-1}}{1-\beta}\left(K(1+g)^{\gamma k-i^{*}}-q(1-\beta)\right)
$$

which is positive for

$$
\frac{K(1+g)^{\gamma k-i^{*}}}{(1-\beta)}>q
$$

so for adopting $i^{*}-t$ more times and then stopping from an out of equilibrium node, $s_{t, k}$, we need:

$$
\frac{K(1+g)^{\gamma k-i^{*}-1}}{(1-\beta)}<q<\frac{K(1+g)^{\gamma k-i^{*}}}{(1-\beta)}
$$

By comparing condition (51) with condition (48) for state $s_{00}$ we can see that the upper bound of (48) is more stringent than the upper bound of (51) and the lower bound of (51) is more stringent than the lower bound of (48). This implies that the condition for non deviating from the strategy profile of increasing asymmetry for firm $A$ from any subgame starting from any possible history of the game is given by:

$$
\frac{K(1+g)^{\gamma k-i^{*}-1}}{(1-\beta)}<q<\frac{K(1+g)^{-i^{*}}}{(1-\beta)}
$$

This interval is not empty if and only if $\gamma k<1$ and given that $k \leq i^{*}$ we need $i^{*}<\frac{1}{\gamma}$.

While firm $A$ is adopting, firm $B$, by deviating would only make 
negative profits before reverting to the candidate equilibrium strategy.

The only interesting deviation for firm $B$ occurs when firm $A$ stops adopting at time $i^{*}$. Therefore for the strategy $F_{i^{*}}$ defined in (14) to be an MPE we want the follower, firm $B$, not to have incentives to deviate and not to adopt the new technology $c_{i^{*}+1}$, once the leader has last adopted technology $c_{i^{*}}$.

This condition has already been derived in the paragraph on absorbing asymmetry and it is given by the condition (31) :

In any state

$$
s_{i^{*}, k}^{A}=\left\{c_{A}=c_{i^{*}}, c_{B}=c_{k}\right\}
$$

for any $k \leq i^{*}$ firm $B$ does not adopt the $\left(i^{*}+1\right)^{t h}$ innovation if and only if:

$$
\frac{K(1+g)^{\gamma i^{*}-i^{*}-1}}{(1-\beta)}<q
$$

Finally by comparing the leader and the follower conditions for the lower bound of the adoption cost, we have that given that (52) has to hold for any $k \leq i^{*}$ then it gives the same lower bound as (53). The adoption costs region for which strategy $F_{i^{*}}$ is a Markov Perfect Equilibrium is then given by:

$$
\frac{K(1+g)^{\gamma i^{*}-i^{*}-1}}{(1-\beta)}<q<\frac{K(1+g)^{-i^{*}}}{(1-\beta)}
$$


which is non empty if and only if

$$
i^{*}<\frac{1}{\gamma}
$$

\section{Bibliography}

- Beath, J., Katsoulacos, Y., Ulph, D., (1994), Strategic $R \& D$ and Innovation, in Current Issues in Industrial Economics, ed Cable, J., Macmillan, London.

- Budd, C., Harris, C., Vickers, J., (1993), "A Model of the Evolution of Duopoly: Does the Asymmetry between Firms Tend to Increase or Decrease?", Review of Economic Studies, 60, 543-573.

- Fudenberg, D., Gilbert, R.,Stiglitz, J., Tirole, J., (1983),"Preemption, Leapfrogging and Competition in Patent Races", European Economic Review, 22, 3-31.

- Fudenberg, D., Tirole, J., (1991), Game Theory, MIT Press, Cambridge, Mass.

- Gilbert, R.J., and Newbery, D.M.G., (1982), "Preemptive Patenting and the Persistence of Monopoly", American Economic Review, 72, 514-526.

- Harris, C., Vickers, J., (1985)," Perfect Equilibrium in a Model of a Race”, Review of Economic Studies, 52,193-209.

- Harris, C., Vickers, J., (1987),"Racing with Uncertainty", Review of 
Economic Studies, 54, 1-21.

- Jovanovic, B., (1997), Learning and Growth in Advances in economics and econometrics: theory and applications, Vol. II, eds Kreps, D., Wallis, K., Cambridge University Press, Cambridge, U.K..

- Kapur, S., (1995), "Technological Diffusion with Social Learning", Journal of Industrial Economics, 43(2), 173-95.

- Kapur, S., (1995), "Markov perfect equilibria in an N-player war of attrition", Economic Letters, 47, 149-154.

- Karshenas, M., Stoneman, P., (1995), "Technological Diffusion" in Handbook of The economics of Innovation and Technological Change, ed Stoneman, P., Basil Blackwell, Oxford.

- Lee, T., Wilde, L., (1980), "Market Structure and Innovation: a Reformulation”, Quarterly Journal of Economics, 94, 429-36.

- Reinganum, J., (1983), "Uncertain Innovation and the Persistence of Monopoly", American Economic Review, 73, 741-748.

- Reinganum, J., (1985), "Innovation and Industry Evolution”, Quarterly Journal of Economics, 100, 81-99.

- Reinganum, J., (1989), The Timing of Innovation:, Research, Development, and Diffusion in Handbook of Industrial Organization, Volume I, eds Schmalensee R., Willig, R.D., Elsevier Science Publisher, Amsterdam. 
- Vickers, J., (1986), "The evolution of Industry Structure when there is a Sequence of Innovations", Journal of Industrial Economics, 35, 1-12.

\section{Footnotes}

1)The idea of Perpetual leapfrogging as a coordination device to avoid aggressive competition is due to a, very appreciated, comment of an anonymous referee.

2)The equilibrium price is given by the unit cost of the non adopter and this is higher when the non adopter is the follower.

3)For a survey see the section on asymmetric models in Reinganum(1989).

4)Note that if $c$ is the initial unit cost at time zero, and if there is adoption by any firm at any period between $t=0$ and $t-1$ then at time $t$ the state of the art technology available for adoption is given by:

$$
c_{t+1}=\frac{c}{(1+g)^{t+1}}
$$

In this case technological clock and time proceed together. If on the contrary at a given time $t$, there have been less adoptions than periods, then:

$$
c_{t+1}=\frac{c}{(1+g)^{\tau+1}}
$$

where $\tau$ is the number of periods in which adoption occurred. In this last case technological and chronological clocks are following different timings.

Given the description of the evolution of the technology, in equilibrium there is either one firm adopting the new innovation or adoption has stopped forever. Any discount factor 
less than one will indeed make the simultaneous postponing of future adoptions, with no change of technology occuring, meaningless.

The characterization of the equilibrium conditions for a date to be an "absorbing technological state" where adoption stops forever and the two clocks start diverging are analysed in the following sections.

5)We are assuming that in the past periods there has been adoption so that technological and chronological clocks are sinchronized. This implies that the costs at time $t$ are:

$$
c_{B}=c_{t-k}=c(1+g)^{-t+k}
$$

given that $B$ has last adopted at time $t-k-1$, and:

$$
c_{A}=c_{t}=c(1+g)^{-t}
$$

given that $A$ has last adopted at time $t-1$.

6)For a more rigorous definition and motivation of the Markov Perfect Equilibrium concept see Fudenberg and Tirole (1991).

7)See the Appendix for the proof of Proposition 3, in Particular Lemma A2.

8)Both $\tilde{V}_{n A}\left(c_{t-k}\right)$ and $\tilde{V}_{n B}\left(c_{t}\right)$, defined in the next paragraph, can be seen as two values of the same function of the last technology $c_{\tau}$, adopted by the opponent :

$\tilde{V}_{n A}\left(c_{t-k}\right)=\tilde{V}_{n}\left(c_{t-k}\right)$ and $\tilde{V}_{n B}\left(c_{t}\right)=\tilde{V}_{n}\left(c_{t}\right)$ where $\tilde{V}_{n}\left(c_{\tau}\right)$ is defined as: 


$$
\tilde{V}_{n}\left(c_{\tau}\right)=\sum_{i=0}^{n-1} \beta^{i}\left[\pi\left(c_{t+i+1}, c_{\tau}\right)-q\right]+\frac{\beta^{n} \pi\left(c_{t+n}, c_{\tau}\right)}{1-\beta}
$$

Note that:

$$
\begin{aligned}
& \sum_{i=0}^{n-1} \beta^{i} \pi\left(c_{t+i+1}, c_{\tau}\right) \\
= & A\left[c_{\tau}^{-\gamma+1} \sum_{i=0}^{n-1} \beta^{i}-c_{\tau}^{-\gamma} c_{t+1}\left(\sum_{i=0}^{n-1} \frac{\beta^{i}}{(1+g)^{i}}\right)\right] \\
= & A\left[\frac{1-\beta^{n}}{1-\beta} c_{\tau}^{-\gamma+1}-c_{\tau}^{-\gamma} c_{t+1} \frac{1-\beta^{n}(1+g)^{-n}}{1-\beta(1+g)^{-1}}\right] \\
= & A\left[\frac{1-\beta^{n}}{1-\beta} c_{\tau}^{-\gamma+1}-c_{\tau}^{-\gamma} c_{t} \frac{(1+g)^{-1}-\beta^{n}(1+g)^{-n-1}}{1-\beta(1+g)^{-1}}\right]
\end{aligned}
$$

so that we can write:

$$
\begin{aligned}
\tilde{V}_{n}\left(c_{\tau}\right)=A & {\left[\frac{1-\beta^{n}}{1-\beta} c_{\tau}^{-\gamma+1}-c_{\tau}^{-\gamma} c_{t} \frac{(1+g)^{-1}-\beta^{n}(1+g)^{-n-1}}{1-\beta(1+g)^{-1}}+\frac{\beta^{n} c_{\tau}^{-\gamma}\left(c_{\tau}-c_{t+n}\right)}{1-\beta}\right] } \\
& -q \frac{1-\beta^{n}}{1-\beta} \\
= & A\left[\frac{1}{1-\beta} c_{\tau}^{-\gamma+1}-c_{\tau}^{-\gamma} c_{t}\left(\frac{(1+g)^{-1}-\beta^{n}(1+g)^{-n-1}}{1-\beta(1+g)^{-1}}+\frac{\beta^{n}(1+g)^{-n}}{1-\beta}\right)\right]- \\
& -q \frac{1-\beta^{n}}{1-\beta} \\
= & A\left[\frac{1}{1-\beta} c_{\tau}^{-\gamma+1}-c_{\tau}^{-\gamma} c_{t}\left(\frac{(1+g)^{-1}(1-\beta)+\beta^{n}(1+g)^{-n}\left(1-(1+g)^{-1}\right)}{\left(1-\beta(1+g)^{-1}\right)(1-\beta)}\right)\right]- \\
& -q \frac{1-\beta^{n}}{1-\beta}
\end{aligned}
$$

9)or 


$$
\begin{gathered}
\beta K(1+g)^{\gamma\left(t^{*}-2\right)-t^{*}+1}-\beta^{2} \frac{K(1+g)^{\gamma\left(t^{*}-1\right)-t^{*}}}{1-\beta}>q(-1+\beta(1-\beta)) \\
\frac{\beta K(1+g)^{\gamma\left(t^{*}-1\right)-t^{*}}\left((1+g)^{-\gamma+1}-\frac{\beta}{1-\beta}\right)}{(-1+\beta(1-\beta))}<q
\end{gathered}
$$

given that $(-1+\beta(1-\beta))<0$.

10)We want to prove that

$$
\frac{\beta}{(1+\beta)}<\frac{1}{(1+g)^{\gamma-1}}
$$

is a sufficient condition for (37) to be more stringent than (41).

From the definition of $t^{*}$ we know that condition (37) implies:

$$
q>\frac{K(1+g)^{\gamma\left(t^{*}\right)-t^{*}-1}}{1-\beta}
$$

therefore if

$$
<\frac{\beta K(1+g)^{\gamma\left(t^{*}-1\right)-t^{*}}\left((1+g)^{-\gamma+1}-\frac{\beta}{1-\beta}\right)}{(-1+\beta(1-\beta))}
$$

condition (41) is satisfied.

Condition (42) can be rewritten as: 


$$
\frac{(1-\beta) \beta(1+g)^{-\gamma+1}\left((1+g)^{-\gamma+1}-\frac{\beta}{1-\beta}\right)}{(-1+\beta(1-\beta))}<1
$$

the denominator is negative so it is sufficient to have that :

$$
(1-\beta) \beta(1+g)^{2(-\gamma+1)}-\beta^{2}(1+g)^{-\gamma+1}>0
$$

or

$$
\frac{\beta}{(1+\beta)}<\frac{1}{(1+g)^{\gamma-1}}
$$

11)It is easy to show that given the description of the strategy $A$ has no power to revert the sequencing order of adoption so that $A$ would not deviate from the equilibrium strategy even if it ends out of the market because $B$ is the last adopter. 